\title{
Fobia social em uma amostra de adolescentes
}

\author{
Gustavo J. Fonseca D'EI Rey \\ Carla Alessandra Pacini \\ Dionísio J. Fontes Chavira \\ Centro de Pesquisas e Tratamentos de Transtornos de Ansiedade - São Paulo
}

\begin{abstract}
Resumo
Este estudo relata a prevalência e o impacto na escolaridade da fobia social em uma amostra de adolescentes da cidade de São Paulo, SP, Brasil. O Inventário de Fobia Social (SPIN) foi administrado em 116 estudantes adolescentes de $5^{\underline{a}}, 6^{\underline{a}}, 7^{\underline{a}}$ e $8^{\underline{a}}$ séries de ambos os sexos. A prevalência da fobia social foi de 7,8\% na amostra de adolescentes, com maior incidência entre estudantes do sexo feminino, com idade entre 12 e 15 . O impacto negativo na escolaridade foi grande, aproximadamente $89 \%$ dos adolescentes com fobia social repetiram o ano na escola ao menos uma vez.

Palavras-chave: fobia social; adolescentes; prevalência; impacto na educação
\end{abstract}

\begin{abstract}
Social phobia in a sample of adolescents. This study reports the prevalence and the impact in the education of social phobia in a sample of adolescents of the city of São Paulo, SP, Brazil. The Social Phobia Inventory (SPIN) was administrated to 116 students of $5^{\text {th }}, 6^{\text {th }}, 7^{\text {th }}$ and $8^{\text {th }}$ grades of both sexes. The prevalence of the social phobia was $7.8 \%$ in the sample of adolescents, with higher incidence among female students, between 12 and 15 years old. The negative impact on the education was great, approximately $89 \%$ of the adolescents with social phobia repeated the year in the school at least one time.

Keywords: social phobia; adolescents; prevalence; impact on education
\end{abstract}

$\mathrm{A}$ ansiedade social surge cedo em nossas vidas, mais precisamente aproximadamente no nono mês de vida é possível observar o medo que algumas crianças têm de estranhos e que costuma desaparecer com o desenvolvimento (Neal, Edelmann, \& Glachan, 2002). Ela está presente durante todo o nosso desenvolvimento e geralmente precede qualquer compromisso social novo ou desconhecido. É vantajoso responder com ansiedade social a certas situações. Para diminuir ou controlar os sintomas da ansiedade social, nós nos preparamos para a situação, tanto na aparência como no comportamento (aulas, festas, encontros amorosos, etc.). Este tipo de ansiedade é considerado normal. Porém, algumas pessoas sentem a ansiedade social de forma tão intensa, que as leva ao sofrimento e a perdas de oportunidades. Elas chegam a abandonar empregos, escola, abdicar de uma vida amorosa, podendo chegar a ponto de viverem completamente isoladas (D’El Rey, 2001).

De acordo com o DSM-IV-TR: Manual Diagnóstico e Estatístico de Transtornos Mentais (American Psychiatric Association, 2002), a fobia social é caracterizada por um medo acentuado e persistente de uma ou mais situações sociais ou de desempenho. A pessoa teme agir de um modo ou mostrar sintomas de ansiedade que lhe sejam humilhantes e embaraçosos, sendo que a exposição à situação social temida provoca uma resposta de ansiedade intensa que pode chegar a um ataque de pânico. A pessoa geralmente evita essas situações ou as suporta com intenso sofrimento. A fobia social apresenta significativa interferência nas rotinas de trabalho, acadêmicas e sociais e/ou sofrimento acentuado.

Os estudos relatam que a fobia social tem seu início na adolescência, segue um curso crônico, trazendo sofrimento e um impacto negativo sobre a vida de seus portadores (Davidson, Hughes, George, \& Blazer, 1993; Magee, Eaton, Wittchen, McGonagle, \& Kessler, 1996; Patel, Knapp, Henderson, \& Baldwin, 2002; Öst, 1987).

Como a fobia social pode ser tratada adequadamente, a identificação do transtorno na adolescência, ou seja, geralmente no seu início, levaria a um menor sofrimento e perdas de oportunidades para seu portador, e diminuiria a chance do aparecimento de comorbidades, estas tão freqüentes na fobia social (Essau, Conradt, \& Petermann, 1999).

Segundo Beidel, Turner e Morris (1999, citados por Vilete, Coutinho, \& Figueira, 2004), na fobia social as manifestações clínicas são basicamente as mesmas nas diferentes faixas 
etárias. As diferenças apenas podem refletir as oportunidades para o engajamento em diferentes tipos de atividades sociais. Por exemplo, uma criança ou adolescente pode não conseguir se esquivar de uma determinada situação social ou de desempenho como faria uma pessoa adulta.

Os estudos internacionais relatam uma prevalência da fobia social em adolescentes entre 1,6 a 7,2\%, dependendo do instrumento diagnóstico utilizado (Essau et al., 1999; Merikangas, Avenevoli, Acharyya, Zhang, \& Angst, 2002; Stein, Fuetsch, Müller, Höfler \& Wittchen, 2001).

Este estudo teve como objetivo verificar a prevalência da fobia social por meio do Inventário de Fobia Social (SPIN) e o impacto na escolaridade (repetência) em uma amostra de estudantes adolescentes da cidade de São Paulo.

\section{Método}

\section{Local e participantes}

O estudo foi realizado em uma escola particular, localizada no bairro da Água Rasa na zona leste da cidade de São Paulo, SP, com aproximadamente 800 alunos. A escola atende estudantes oriundos de famílias de classe socioeconômica média.

Foram entrevistados 116 adolescentes, sendo 49 (42,2\%) do sexo masculino e $67(57,8 \%)$ do sexo feminino. Os participantes eram estudantes de $5^{\underline{a}}$ a $8^{\underline{a}}$ séries da referida escola, com idades entre 10 e 17 anos, com idade média de 12,5 anos. As características dos adolescentes pesquisados encontramse detalhadas na Tabela 1.

Tabela 1

\begin{tabular}{|c|c|c|}
\hline Características & $\mathrm{N}$ & $\%$ \\
\hline \multicolumn{3}{|l|}{ Sexo } \\
\hline Masculino & 49 & 42,2 \\
\hline Feminino & 67 & 57,8 \\
\hline \multicolumn{3}{|l|}{ Idade } \\
\hline $10-11$ & 34 & 29,3 \\
\hline $12-13$ & 53 & 45,7 \\
\hline $14-15$ & 27 & 23,3 \\
\hline $16-17$ & 2 & 1,7 \\
\hline \multicolumn{3}{|l|}{ Série } \\
\hline $5^{\underline{a}}$ & 35 & 30,2 \\
\hline $6^{\mathrm{a}}$ & 31 & 26,7 \\
\hline $7^{\underline{a}}$ & 27 & 23,3 \\
\hline $8^{\mathrm{a}}$ & 23 & 19,8 \\
\hline \multicolumn{3}{|l|}{ Repetência } \\
\hline Nenhuma & 105 & 90,5 \\
\hline $1 \mathrm{vez}$ & 9 & 7,8 \\
\hline 2 vezes ou mais & 2 & 1,7 \\
\hline
\end{tabular}

\section{Instrumento de avaliação}

O Inventário de Fobia Social (SPIN) é um instrumento que consiste de 17 itens, que abrangem 3 critérios importantes que definem o quadro clínico da fobia social, ou seja, o medo, a evitação das situações e os sintomas somáticos. O inventário engloba tanto situações de interação social quanto de desempenho. Para cada item do questionário, solicitase ao indivíduo que indique o quanto as situações o incomodaram na última semana, devendo marcar uma entre cinco opções, que variam entre De forma alguma a Extremamente. A pontuação para cada uma das opções varia de 0 a 4 , e a pontuação total do inventário varia de 0 a 68. O SPIN apresenta uma grande utilidade diagnóstica como um instrumento de rastreamento (screening). Ele é capaz de discriminar bem pessoas com e sem fobia social. Escores de 19 pontos ou mais indicam a presença de sintomas compatíveis com o diagnóstico de fobia social. Outro fator positivo do SPIN é sua fácil e rápida aplicação (poucos minutos) e também a rápida apuração de seu escore (Connor et al., 2000). No Brasil, existe um estudo de confiabilidade da versão em português do Inventário de Fobia Social (SPIN), demonstrando que ele apresenta resultados de boa confiabilidade, semelhantes aos da versão original em inglês (Vilete et al., 2004).

\section{Procedimentos}

Aleatoriamente foram escolhidas 4 salas de aula (uma sala de cada série $-5^{\underline{a}}$ a $8^{\underline{a}}$ ) para a aplicação do presente estudo. Após uma breve explicação sobre os objetivos da pesquisa, foi solicitado aos estudantes que preenchessem um questionário com os dados sócio-demográficos e em seguida respondessem as questões do Inventário de Fobia Social (SPIN). Os autores deste estudo estiveram presentes durante a aplicação, para o esclarecimento de qualquer dúvida que os adolescentes pudessem ter durante o preenchimento do questionário e do SPIN. A aplicação foi realizada na própria sala de aula em uma única sessão.

\section{Questões éticas}

Após a aceitação por parte da escola, como cuidado ético foi enviado aos pais e responsáveis um termo livre, solicitando a autorização para que seus filhos participassem da presente pesquisa. Este termo foi encaminhado por intermédio do próprio aluno. No termo, os pais e responsáveis foram orientados a respeito do objetivo do estudo, do tipo de participação requerida, bem como da ausência de prejuízos decorrentes da não-participação. Os estudantes adolescentes que entregaram o termo de autorização cujos pais permitiram sua participação na pesquisa foram elegíveis para o estudo. Aos adolescentes participantes foi assegurado o caráter confidencial da pesquisa e foi informado que suas respostas não influenciariam de forma alguma em suas notas ou desempenho escolar. Dezenove por cento dos alunos que poderiam fazer parte deste estudo, não apresentaram o termo de consentimento assinado pelos responsáveis no dia da aplicação do SPIN, razão pela qual não participaram da pesquisa. Os adolescentes que apresentaram sintomas compatíveis com o diagnóstico de fobia social foram encaminhados para tratamento.

\section{Resultados}

De acordo com o Inventário de Fobia Social (SPIN), nove alunos (ou 7,8\% dos 116 sujeitos do estudo) obtiveram esco- 
re igual ou superior a 19 pontos no referido inventário, pontuação esta que indica presença de sintomas compatíveis com o diagnóstico de fobia social. Os escores do SPIN desses nove estudantes adolescentes variaram entre 21 a 54 pontos, com média de 34,1 pontos $(D P=10,8)$.

Dos 49 participantes do sexo masculino, quatro $(8,1 \%)$ apresentaram pontuação que indica possível manifestação de fobia social e, das 67 estudantes do sexo feminino, cinco $(7,4 \%)$ apresentaram pontuação de fobia social.

A grande maioria dos estudantes com sintomas fóbicos sociais tinha entre 12 e 15 anos (66,7\%). A maior concentração ocorreu nos adolescentes da $6^{\underline{\underline{a}}}$ e $7^{\underline{\underline{a}}}$ séries $(66,7 \%)$. Aproximadamente todos os adolescentes com sintomas compatíveis com o diagnóstico de fobia social repetiram pelo menos uma vez de ano na escola (88,9\%). A Tabela 2 apresenta as características dos estudantes adolescentes com fobia social.

Tabela 2

Adolescentes com fobia social segundo o SPIN $(N=9)$

\begin{tabular}{|c|c|c|}
\hline Características & $\mathrm{N}$ & $\%$ (I.C.) \\
\hline Sexo & & \\
\hline Masculino & 4 & $44,4(42,8-46,0)$ \\
\hline Feminino & 5 & $55,6(54,0-57,2)$ \\
\hline \multicolumn{3}{|l|}{ Idade } \\
\hline $10-11$ & 1 & $11,1 \quad(9,0-13,2)$ \\
\hline $12-13$ & 3 & $33,3(31,2-35,4)$ \\
\hline $14-15$ & 3 & $33,3(31,2-35,4)$ \\
\hline $16-17$ & 2 & $22,2(20,1-24,3)$ \\
\hline \multicolumn{3}{|l|}{ Série } \\
\hline $5^{-\mathbf{a}}$ & 1 & $11,1 \quad(9,0-13,2)$ \\
\hline $6^{\mathrm{a}}$ & 3 & $33,3(31,2-35,4)$ \\
\hline $7^{\mathrm{a}}$ & 3 & $33,3(31,2-35,4)$ \\
\hline $8^{\underline{a}}$ & 2 & $22,2(20,1-24,3)$ \\
\hline \multicolumn{3}{|l|}{ Repetência } \\
\hline Nenhuma & 1 & $11,1 \quad(5,3-16,9)$ \\
\hline $1 \mathrm{vez}$ & 6 & $66,7(60,9-72,5)$ \\
\hline 2 vezes ou mais & 2 & $22,2(16,4-28,0)$ \\
\hline
\end{tabular}

I.C. = Intervalo de Confiança

\section{Discussão}

Em nosso estudo, encontramos uma prevalência similar $(7,8 \%)$ a outros estudos que avaliaram a presença da fobia social em adolescentes (Essau et al., 1999; Merikangas et al., 2002; Stein et al., 2001). Embora estes estudos internacionais tenham usado metodologias diferentes do nosso, podemos dizer que em virtude de encontrarmos uma prevalência similar, isto sugere que o Inventário de Fobia Social (SPIN) mostrou-se um bom instrumento para rastreamento de sintomas compatíveis com o diagnóstico de fobia social em adolescentes no Brasil. Cabe lembrar que o SPIN faz apenas o rastreamento dos casos que podem apresentar fobia social, precisando de confirmações em entrevistas futuras, pois o próprio DSM-IV-TR (American Psychiatric Association, 2002) solicita critérios para o diagnóstico de fobia social em meno- res de 18 anos que não estão presentes no SPIN, como é o caso da duração dos sintomas.

A maioria dos adolescentes com sintomas compatíveis com o diagnóstico de fobia social em nosso estudo tinha entre 12 e 15 anos, idade crítica para o desenvolvimento pessoal, pois é nessa faixa etária que desenvolvemos uma série de habilidades sociais, como o comportamento de auto-afirmação, habilidades de paquera, etc. Parece provável que esses adolescentes, sem tratamento adequado, não conseguirão atingir um nível esperado de funcionamento. Isto nos faz pensar sobre o impacto negativo destes sintomas na vida destes adolescentes, como afirmam Neal et al. (2002), Chartier, Walker e Stein (2001) e Wittchen e Beloch (1996).

A fobia social com freqüência leva as pessoas acometidas a uma limitação funcional importante e que pode ser medida nas áreas educacional, profissional e social. Em nosso estudo, avaliamos a impacto da fobia social sobre a escolaridade dos adolescentes. Dos nove adolescentes com sintomas compatíveis com a fobia social, oito haviam repetido ao menos uma vez o ano na escola (seis uma vez, e dois, duas vezes ou mais). Em comparação aos adolescentes sem sintomas fóbicos sociais, aproximadamente apenas 3\% haviam repetido o ano na escola (todos apenas uma vez). Provavelmente o medo da interação social com seus pares na escola limita o desempenho dos adolescentes com fobia social, restringindo suas capacidades para a realização de seu potencial educacional. Os dados encontrados neste nosso estudo em relação à repetência nos adolescentes com possível fobia social corroboram a afirmação de Van Ameringen, Mancini e Farvolden (2003) e de Davidson et al. (1993), ou seja, pessoas com esse transtorno invariavelmente apresentam histórias escolares com pelo menos uma repetência escolar.

Van Ameringen et al. (2003) mencionam que as pessoas acometidas pela fobia social apresentam um grande risco para deixarem a escola prematuramente. Perguntamo-nos, quantos destes adolescentes em nosso estudo, poderão deixar os estudos prematuramente num futuro próximo, sem o tratamento adequado? Segundo estudos (Berg, 1992; Kessler, Foster, Sanuders, \& Stang, 1995; Last \& Strauss, 1990; Nardi, 2000), as pessoas acometidas por fobia apresentam uma baixa realização educacional, com os anos gastos na escola reduzidos em média de um a dois anos.

Podemos concluir que sintomas compatíveis com o diagnóstico de fobia social são prevalentes em adolescentes, podendo acarretar conseqüências negativas para a escolaridade. A maioria dos adolescentes com sintomas fóbicos sociais havia repetido pelo menos uma vez o ano na escola.

O Inventário de Fobia Social (SPIN) se mostrou adequado no rastreamento da fobia social em adolescentes. Outros fatores também apontam para sua divulgação entre os pesquisadores e clínicos no Brasil, ou seja, seus itens abrangem três critérios importantes que definem o quadro clínico da fobia social: o medo, a evitação das situações e os sintomas somáticos. O SPIN requer poucos minutos para seu preenchimento e também uma rápida contagem de seu escore. Porém, deve-se ter em mente que a versão original em inglês foi 
construída para a avaliação da fobia social em adultos, e que a compreensão de seus itens pode apresentar algum grau de dificuldade para menores de 18 anos, principalmente crianças.

Os resultados deste estudo sugerem que devemos identificar e tratar os casos de fobia social nos adolescentes, pois desta maneira podemos minimizar senão todas, ao menos as principais seqüelas que o transtorno deixa na vida de seu portador, visando desta maneira reduzir o alto preço que o fóbico social tem de pagar pelas limitações impostas em seu funcionamento educacional, social e ocupacional.

Sugerimos que outros estudos em relação à fobia social na adolescência sejam realizados com grupos maiores para melhor confirmação dos dados encontrados.

\section{Referências}

American Psychiatric Association (2002). DSM-IV-TR: Manual Diagnóstico e Estatístico de Transtornos Mentais ( $4^{\mathrm{a}}$ ed., texto revisado). Porto Alegre: Artmed.

Berg, I. (1992). Absence from school and mental health. British Journal of Psychiatry, 161, 154-166.

Chartier, M. J., Walker, J. R., \& Stein, M. B. (2001). Social phobia and potential childhood risk factors in a community sample. Psychological Medicine, 31, 307-315.

Connor, K. M., Davidson, J. R. T., Churchill, L. E., Sherwood, A., Foa, E., \& Weisler, R. H. (2000). Psychometric properties of the social phobia inventory (SPIN): a new self-rating scale. British Journal of Psychiatry, 176, 379-386.

Davidson, J. T. R., Hughes, D. L., George, L. K., \& Blazer, D. G. (1993). The epidemiology of social phobia: findings from the epidemiological catchment area study. Psychological Medicine, 23, 709-718.

D’El Rey, G. J. F. (2001). Fobia social: mais do que uma simples timidez. Arquivos de Ciências da Saúde da Unipar, 5, 273-276.

Essau, C. A., Conradt. J., \& Petermann, F. (1999). Frequency and comorbidity of social phobia and social fears in adolescents. Behaviour Research and Therapy, 37, 831-843.
Kessler, R. C., Foster, M. P. H., Sanuders, W. B., \& Stang, P. E. (1995). Social consequences of psychiatry disorders I: educational attainment. American Journal of Psychiatry, 152, 1026-1032.

Last, C. G., \& Strauss, C. C. (1990). School refusal in anxiety disordered children and adolescents. Journal of the American Academy of Child and Adolescent Psychiatry, 29, 31-35.

Magee, W. J., Eaton, W. W., Wittchen, H-U., McGonagle, K. A., \& Kessler, R. C. (1996). Agoraphobia, simple phobia and social phobia in the National Comorbidity Survey. Archives of General Psychiatry, 53, 159-168.

Merikangas, K. R., Avenevoli, S., Acharyya, S., Zhang, H., \& Angst, J. (2002). The spectrum of social phobia in the Zurich cohort study of adolescents. Biological Psychiatry, 51, 81-91.

Nardi, A. E. (2000). Complicações e limitações. In Transtorno de ansiedade social: fobia social - a timidez patológica (pp. 51-57). Rio de Janeiro: Medsi.

Neal, J. A., Edelmann, R. J., \& Glachan, M. (2002). Behavioural inhibition and symptoms of anxiety and depression: is there a specific relationship with social phobia? British Journal of Clinical Psychology, 41, 361-374.

Öst, L. G. (1987). Age of onset of different phobias. Journal of Abnormal Psychology, 96, 223-229.

Patel, A., Knapp, M., Henderson, J., \& Baldwin, D. (2002). The economic consequences of social phobia. Journal of Affective Disorders, 68, 221-233.

Stein, M. B., Fuetsch, M., Müller, N., Höfler, M., \& Wittchen, H-U. (2001). Social anxiety disorder and the risk of depression: a prospective community study of adolescents. Archives of General Psychiatry, 58, 251-256.

Van Ameringen, M., Mancini, C., \& Farvolden, P. (2003). The impact of anxiety disorders on educational achievement. Journal of Anxiety Disorders, 17, 561-571.

Vilete, L. M. P., Coutinho, E. S. F., \& Figueira, I. L. V. (2004). Confiabilidade da versão em português do inventário de fobia social (SPIN) entre adolescentes estudantes do município do Rio de Janeiro. Cadernos de Saúde Pública, 20, 89-99.

Wittchen, H-U., \& Beloch, E. (1996). The impact of social phobia on quality of life. International Clinical Psychopharmacology, 11, 15-23.

Gustavo J. Fonseca D’El Rey, psicólogo e especialista em Psicologia Hospitalar pelo Instituto Central do Hospital das Clínicas da Faculdade de Medicina da Universidade de São Paulo, é coordenador do Programa de Fobia Social do Centro de Pesquisas e Tratamentos de Transtornos de Ansiedade, em São Paulo, SP. Endereço para correspondência: Rua Bom Jesus, 274-B; São Paulo, SP; CEP 03344-000. Tel./Fax: (11) 6965-5163. Email: g.delrey@bol.com.br

Carla Alessandra Pacini, pedagoga com habilitação em Administração Escolar pela Faculdade Campos Salles, é pesquisadora no Programa de Fobia Social do Centro de Pesquisas e Tratamentos de Transtornos de Ansiedade, em São Paulo, SP.

Dionísio J. Fontes Chavira, doutor em Psiquiatria Clínica pelo Instituto de Psiquiatria do Maudsley Hospital, de Londres (Reino Unido), é pesquisador no Programa de Fobia Social do Centro de Pesquisas e Tratamentos de Transtornos de Ansiedade, em São Paulo, SP. 